\title{
Bianchi type I bulk viscous barotropic fluid cosmological model with varying $\Lambda$ and functional relation on Hubble parameter in self-creation theory of gravitation
}

\author{
Mukunda Sudam Borkar ${ }^{1}$, Nilesh Kawaduji Ashtankar ${ }^{2}$ \\ ${ }^{1}$ P. G. Department of Mathematics, Mahatma Jyotiba Phule Educational Campus, Amravati Road, R T M Nagpur University, Nagpur, India \\ ${ }^{2}$ J.D. College of Engineering, At: Khandala, Post: Valni, Katol Road, Nagpur- 441501 (India)
}

\section{Email address:}

borkar.mukund@rediffmail.com (M. S. Borkar), ashtankarnilesh1@gmail.com (N. K. Ashtankar)

\section{To cite this article:}

Mukunda Sudam Borkar, Nilesh Kawaduji Ashtankar. Bianchi Type I Bulk Viscous Barotropic Fluid Cosmological Model with Varying $\boldsymbol{\Lambda}$ and Functional Relation on Hubble Parameter in Self-Creation Theory of Gravitation. American Journal of Modern Physics. Vol. 2, No. 5, 2013, pp. 264-269. doi: 10.11648/j.ajmp.20130205.15

\begin{abstract}
We have investigated Bianchi type I bulk viscous barotropic fluid cosmological model with varying $\Lambda$ and functional relation on Hubble parameter in self-creation theory of gravitation. It is observed that the scalar function $\phi(t)$ affected the physical parameters of the universe and our model may have more than three spatial-dimensions in the beginning which is a theoretical evidence pointed out in the geometry of universe. The propose variation law for Hubble parameter leads cosmological model which is dominated by decelerating phase at initial stage and at later stage, the universe has accelerating phase and the universe expanding exponentially with acceleration supporting the observational data. Also other geometrical and physical properties of the model have been evaluated.
\end{abstract}

Keywords: Self-Creation Gravitational Theory, Cosmological Model, Cosmological Constant and Hubble Parameter

\section{Introduction}

Since Einstein himself pointed out that his general theory of gravitation does not account satisfactorily for the inertial properties of matter. i.e., Mach's principle is not substantiated by general relativity, there have been some interesting attempts to generalize the general theory of gravitation by incorporating Mach's principle and other desired features which were lacking in the original theory and hence Barber ${ }^{[1]}$ modified it by coupling the scalar field with the energy momentum tensor so that the Mach's principle is substantially accommodate by the theory. The modification of Einstein field equations which are taken into consideration by Barber ${ }^{[1]}$ in his self-creation theory are,

$$
G_{i j}=R_{i j}-\frac{1}{2} R g_{i j}=-\frac{8 \pi \lambda T_{i j}}{\phi}
$$

where, $\phi(t)$ is the Barber scalar function of $t, T_{i j}$ is the energy momentum tensor, $G_{i j}$ is an Einstein tensor and $\lambda$ is the coupling constant.

Barber's ${ }^{[1]}$ theory is a variable G-theory and predicts local effects, which are within the observational limits. In it, the Newtonian gravitational parameter $\mathrm{G}$ is not a constant but a function of time parameter $t$. Also the scalar field $\phi(t)$ does not gravitate directly but divides the matter tensor acting as a reciprocal gravitational constant, which is not the case in Einstein theory of gravitation. This theory is capable of verification or falsification. It can be done by observing the behavior of both bodies of degenerate matter and photons. An observation of anomalous precessions in the orbits of pulsars about central masses and an accurate determination of the deflection of light and radio waves passing close to the sun would verify or falsify such theory and determines $\lambda$. The theory predicts the same precession of the perihelia of the planets as that of general relativity and in that respect agrees with observation to within $1 \%$.

Pimental $^{[2]}$ and Soleng ${ }^{[3]}$ have presented the Robertson Walker solutions in self-creation theory of gravitation by using power law relation between the expansion factor of the universe and the scalar field. Reddy and Venkateswarlu ${ }^{[4]}$ have obtained spatially homogeneous and anisotropic Bianchi type- $\mathrm{VI}_{0}$ cosmological models in Barber's self-creation theory of gravitation in both vacuum as well as in presence of perfect fluid with pressure equal to energy density. Venkateswarlu and Reddy ${ }^{[5]}$ have also got spatially homogeneous and anisotropic Bianchi type-I cosmological macro models when the source of gravitational field is a 
perfect fluid. Bianchi type-II and III models in self-creation cosmology have been deduced by Shanti and Rao ${ }^{[6]}$. V.U.M. Rao and Y.V.S.S. Sanyasiraju ${ }^{[7]}$ have discussed Bianchi type VIII and IX in zero mass scalar fields and self-creation cosmology. S. Ram and C. P. Sigh ${ }^{[8]}$ have studied early universe in self-creation cosmology. A. Pradhan and H. R. Pandey ${ }^{[9]}$ have studied bulk viscous cosmological models in Barber's second self-creation theory. Recently the plane and axially symmetric cosmological models and also Bianchi type-I, II, VIII and IX string cosmological models in self-creation theory of gravitation have been deduced by many researchers ${ }^{[10-18]}$.

In this note, we have taken-up the study of Bianchi type I bulk viscous barotropic fluid cosmological model with varying $\Lambda$ and functional relation on Hubble parameter in self-creation theory of gravitation and evaluated the nature of the universe. It is seen that the Barber's scalar function $\phi(t)$ affected the physical parameters of the universe and the universe may have more than three spatial-dimensions in the beginning which is the theoretical evidence pointed out in the geometry of the universe. The propose variation law for Hubble parameter leads our cosmological model which is dominated by decelerating phase at initial stage and at later stage, the universe has accelerating phase and the universe expanding with acceleration supporting the observational data ${ }^{[19-22]}$. Also other geometrical and physical properties of the model have been evaluated.

\section{The Metric and Field Equations}

Consider Bianchi Type I metric

$$
d s^{2}=-d t^{2}+A^{2}(t) d x^{2}+B^{2}(t) d y^{2}+C^{2}(t) d z^{2}
$$

The matter filled with bulk viscous barotropic fluid with energy-momentum tensor given by,

$$
T_{i}^{j}=(\rho+\bar{p}) v_{i} v^{j}+\bar{p} g_{i}^{j}
$$

where $\bar{p}$ is the effective pressure for viscosity and it is

$$
\bar{p}=p-\zeta v_{\mid i}^{i}
$$

(Here and hereafter the notation '|' stands for the covariant derivative.)

in which $p$ is the isotropic pressure, $\rho$ is energy density, $\zeta$ is the coefficient of bulk viscosity and four velocity vector $v_{i}$ is given by,

$$
v_{i} v^{j}=-1
$$

The field equations in Barber self-creation theory with time varying cosmological constant $\Lambda(t)$ are given by

$$
R_{i}^{j}-\frac{1}{2} R g_{i}^{j}=-8 \pi \phi^{-1} T_{i}^{j}+\Lambda(t) g_{i}^{j}
$$

and

$$
\phi=\frac{8}{3} \pi \lambda T
$$

where, $\phi(t)$ is the Barber's scalar function of $t, \lambda$ is the coupling constant and $0 \leq|\lambda| \leq 10^{-1}, \quad \phi$ is the invariant $\mathrm{d}$ 'Alembertian, $\mathrm{T}$ is the trace energy-momentum tensor.

Assume the comoving coordinate system, then the field equations (5) and (6) for the metric (1) takes the form,

$$
\begin{aligned}
- & \frac{\ddot{B}}{B}-\frac{\ddot{C}}{C}-\frac{\dot{B} \dot{C}}{B C}=8 \pi \phi^{-1} \bar{p}-\Lambda \\
- & \frac{\ddot{A}}{A}-\frac{\ddot{C}}{C}-\frac{\dot{A} \dot{C}}{A C}=8 \pi \phi^{-1} \bar{p}-\Lambda \\
- & \frac{\ddot{A}}{A}-\frac{\ddot{B}}{B}-\frac{\dot{A} \dot{B}}{A B}=8 \pi \phi^{-1} \bar{p}-\Lambda \\
& \frac{\dot{A} \dot{B}}{A B}+\frac{\dot{B} \dot{C}}{B C}+\frac{\dot{A} \dot{C}}{A C}=8 \pi \phi^{-1} \rho+\Lambda \\
\ddot{\phi}+ & \dot{\phi}\left[\frac{\dot{A}}{A}+\frac{\dot{B}}{B}+\frac{\dot{C}}{C}\right]+\frac{8}{3} \pi \lambda(3 \bar{p}-\rho)=0
\end{aligned}
$$

respectively.

Denote, $\dot{A}=\frac{\partial A}{\partial t}, \ddot{A}=\frac{\partial^{2} A}{\partial t^{2}}, \dot{B}=\frac{\partial B}{\partial t}, \ddot{B}=\frac{\partial^{2} B}{\partial t^{2}}$,

$\dot{\phi}=\frac{\partial \phi}{\partial t}, \ddot{\phi}=\frac{\partial^{2} \phi}{\partial t^{2}}$ etc.

\section{Solution of the Field Equations}

There is a system of five differential equations (7-11) in seven unknowns $A, B, C, p, \rho, \Lambda$ and $\phi$. In order to have solution, we assume two conditions. As non-vacuum component of matter obeys the equation of state, first we assume the condition of equation of state as

$$
p=\omega \rho, 0 \leq \omega \leq 1
$$

From equations (7-9), we write.

$$
\begin{aligned}
& \frac{\ddot{A}}{A}-\frac{\ddot{B}}{B}+\frac{\dot{C}}{C}\left(\frac{\dot{A}}{A}-\frac{\dot{B}}{B}\right)=0 \\
& \frac{\ddot{B}}{B}-\frac{\ddot{C}}{C}+\frac{\dot{A}}{A}\left(\frac{\dot{B}}{B}-\frac{\dot{C}}{C}\right)=0
\end{aligned}
$$

from which, we arrived at

$$
\begin{aligned}
& \frac{\dot{A}}{A}-\frac{\dot{B}}{B}=\frac{k_{1}}{A B C} \\
& \frac{\dot{B}}{B}-\frac{\dot{C}}{C}=\frac{k_{2}}{A B C}
\end{aligned}
$$

where, $k_{1}$ and $k_{2}$ are constants of integration.

The spatial volume is $V=A B C$ and an average scale factor $\mathrm{R}$ is define as $R^{3}=A B C$.

The functional relation on Hubble parameter $H$ with deceleration parameter $q$ and $\mathrm{R}$ are given by,

$$
\begin{array}{r}
H=\frac{\dot{R}}{R}=\frac{1}{3}\left(\frac{\dot{A}}{A}+\frac{\dot{B}}{B}+\frac{\dot{C}}{C}\right) \\
q=-\frac{\ddot{R}}{R H^{2}}
\end{array}
$$

And

Using this relation (17), the equations (15) and (16) yield the following equations, 


$$
\begin{aligned}
& \frac{\dot{A}}{A}=\frac{\dot{R}}{R}+\frac{2 k_{1}+k_{2}}{3 R^{3}} \\
& \frac{\dot{B}}{B}=\frac{\dot{R}}{R}+\frac{k_{2}-k_{1}}{3 R^{3}} \\
& \frac{\dot{C}}{C}=\frac{\dot{R}}{R}-\frac{\left(k_{1}+2 k_{2}\right)}{3 R^{3}}
\end{aligned}
$$

We consider

$$
H(R)=a\left(R^{-n}+1\right) a>0, n>1
$$

For this choice of Hubble parameter $H$, the deceleration parameter $q$ turns out to be

$$
q=\frac{n}{R^{n}+1}-1
$$

It is observed that, when $R=0, q=n-1>0, q=0$ for $R^{n}=n-1$ and for $R^{n}>n-1, q<0$.

Integrating equation (22), we get

$$
R^{n}=e^{n a(t+d)}-1
$$

where $d$ is a constant of integration.

By assuming $R=0$ for $t=0$, we obtained $d=0$ so that,

$$
R^{n}=e^{n a t}-1
$$

Using this equation (25), from equations (19-21), we calculated $A(t), B(t)$ and $C(t)$

$$
\begin{aligned}
& A=d_{1}\left(e^{n a t}-1\right)^{\frac{1}{n}} e^{\frac{2 k_{1}+k_{2}}{3}\left[-\frac{1}{3 a} e^{-3 a t} 2 F 1\left(\frac{3}{n}, \frac{3}{n} ; 1+\frac{3}{n} ; e^{-n a t}\right)\right]} \\
& B=d_{2}\left(e^{n a t}-1\right)^{\frac{1}{n}} e^{\frac{k_{2}-k_{1}}{3}\left[-\frac{1}{3 a} e^{-3 a t} 2 F 1\left(\frac{3}{n}, \frac{3}{n} ; 1+\frac{3}{n} ; e^{-n a t}\right)\right]} \\
& C=d_{3}\left(e^{n a t}-1\right)^{\frac{1}{n}} e^{\frac{k_{1}+2 k_{2}}{3}\left[-\frac{1}{3 a} e^{-3 a t} 2 F 1\left(\frac{3}{n}, \frac{3}{n} ; 1+\frac{3}{n} ; e^{-n a t}\right)\right]}
\end{aligned}
$$

in which $d_{1}, d_{2}, d_{3}$ are constant of integrations.

Therefore the metric (1), goes over to

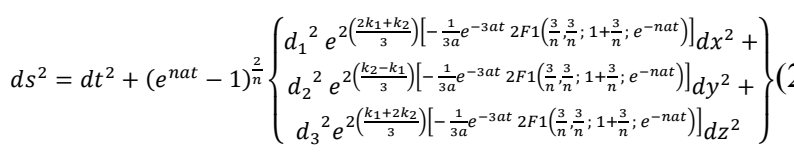

In this metric, $2 F 1\left(\frac{3}{n}, \frac{3}{n} ; 1+\frac{3}{n} ; e^{-n a t}\right)$ is the Gauss hypergeometric function.

It is seen that,

$2 F 1 \rightarrow \infty$ as $t \rightarrow 0$

and $2 F 1=1$ as $t \rightarrow \infty$, for all $n>1$.

\section{Geometrical and Physical Parameters and their Significance}

The components of shear tensor $\sigma_{j}^{i}$ for the model (29) are

$$
\sigma_{1}^{1}=\left(\frac{\dot{A}}{A}-\frac{\dot{R}}{R}\right)=\frac{2 k_{1}+k_{2}}{3 R^{3}}
$$

$$
\begin{gathered}
\sigma_{2}^{2}=\left(\frac{\dot{B}}{B}-\frac{\dot{R}}{R}\right)=\frac{k_{2}-k_{1}}{3 R^{3}} \\
\sigma_{3}^{3}=\left(\frac{\dot{C}}{C}-\frac{\dot{R}}{R}\right)=-\frac{\left(k_{1}+2 k_{2}\right)}{3 R^{3}} \\
\sigma_{4}^{4}=0
\end{gathered}
$$

and the shear $\sigma$ is

$$
\sigma^{2}=\frac{k_{1}{ }^{2}+k_{2}{ }^{2}+k_{1} k_{2}}{3 R^{6}}=\frac{k^{2}}{3 R^{6}}
$$

where,

$$
k^{2}=k_{1}{ }^{2}+k_{2}{ }^{2}+k_{1} k_{2}
$$

Equations (7-11) in terms of $H, \sigma$, and $q$ can be written as

$$
\begin{aligned}
& 8 \pi \phi^{-1} \bar{p}-\Lambda=H^{2}(2 q-1)-\sigma^{2} \\
& 8 \pi \phi^{-1} \rho+\Lambda=3 H^{2}-\sigma^{2} \\
& \ddot{\phi}+3 H \dot{\phi}+\frac{8}{3} \pi \lambda(3 \bar{p}-\rho)=0
\end{aligned}
$$

and for conservation of matter, from equation (2), we write

$$
8 \pi\left[\dot{\rho}+3(\rho+\bar{p}) \frac{\dot{R}}{R}-\rho \phi^{-1} \dot{\phi}\right]+\dot{\Lambda} \phi=0
$$

Using equation (25), we write the deceleration parameter $q$ from equation (23) and shear $\sigma$, from equation (34) as,

$$
\begin{aligned}
q & =\frac{n}{e^{n a t}}-1 \\
\sigma & =\frac{k}{\sqrt{3}\left(e^{n a t}-1\right)^{\frac{3}{n}}}
\end{aligned}
$$

The expansion $\left(\theta=v_{\mid i}^{i}\right)$ is given by

$$
\theta=3 H=\frac{3 a}{1-e^{-n a t}}
$$

On adding equations (36) and (37), we get,

$8 \pi \phi^{-1}(\bar{p}+\rho)=8 \pi \phi^{-1}(p-\zeta \theta+\rho)=2 H^{2}(q+1)-2 \sigma^{2}($

from which, we have calculated the values of $\rho$ and $p$

(using equation (12) and equations (40-42)) as,

$$
\rho=\frac{1}{8 \pi(\omega+1)}\left[\frac{2 n a^{2} e^{n a t}}{\left(e^{n a t}-1\right)^{2}}-\frac{2 k^{2}}{3\left(e^{n a t}-1\right)^{\frac{6}{n}}}\right] \phi(t)+\frac{3 \zeta a}{(\omega+1)\left(1-e^{-n a t}\right)}
$$

$p=\frac{\omega}{8 \pi(\omega+1)}\left[\frac{2 n a^{2} e^{n a t}}{\left(e^{n a t}-1\right)^{2}}-\frac{2 k^{2}}{3\left(e^{n a t}-1\right)^{\frac{6}{n}}}\right] \phi(t)+\frac{3 \omega \zeta a}{(\omega+1)\left(1-e^{-n a t}\right)}$

From equation (10), we have

$$
\Lambda=\frac{a^{2} e^{n a t}}{\left(e^{n a t}-1\right)^{2}}\left[3 e^{n a t}-\frac{2 n}{\omega+1}\right]+\frac{k^{2}(1-\omega)}{3\left(e^{n a t}-1\right)^{\frac{6}{n}}(\omega+1)}-\frac{24 \pi a \zeta}{(\omega+1)\left(1-e^{-n a t}\right)} \phi^{-1}
$$

Using these values of $\rho, p$ and $\Lambda$ in equation (38), after lengthy but straightforward calculations, we yield

$$
\ddot{\phi}+\frac{3 a}{\left(1-e^{-n a t}\right)} \dot{\phi}+\left[\alpha_{1} \frac{e^{n a t}}{\left(e^{n a t}-1\right)^{2}}-\beta_{1} \frac{1}{\left(e^{n a t}-1\right)^{\frac{6}{n}}}\right] \phi=\frac{\beta_{2}-\alpha_{2}}{1-e^{-n a t}}
$$


in which $\alpha_{1}, \alpha_{2}, \beta_{1}$ and $\beta_{2}$ are constants given by

$\alpha_{1}=\frac{2 \lambda n a^{2}(3 \omega-1)}{3(\omega+1)}, \alpha_{2}=\frac{8 \pi \lambda \zeta a(3 \omega-1)}{\omega+1}, \beta_{1}=\frac{2 \lambda k^{2}(3 \omega-1)}{9(\omega+1)}$,

$\beta_{2}=24 \pi \lambda \zeta a$

For simplicity, we assume, the coefficient of $\phi$ in (47) is zero, then

$$
\beta_{1}=\alpha_{1}\left(e^{n a t}-1\right)^{\frac{6-2 n}{n}} e^{n a t}
$$

Then the above differential equation (47) becomes

$$
\ddot{\phi}+\frac{3 a e^{n a t}}{\left(e^{n a t}-1\right)} \dot{\phi}=\frac{\alpha e^{n a t}}{\left(e^{n a t}-1\right)}
$$

(where $=\left(\beta_{2}-\alpha_{2}\right)$, a constant)

which after straightforward calculations, yield

$$
\phi(t)=\frac{1}{3 a}\left[\alpha t-c_{1} e^{-3 a t} 2 F 1\left(\frac{3}{n}, \frac{3}{n} ; 1+\frac{3}{n} ; e^{-n a t}\right)\right]+c_{2}
$$

It is seen that, the Barber's scalar function $\phi(t)$ is appeared in the physical quantities like $\rho, p$ and $\Lambda$. It is also observed that the Gauss hypergeometric function $2 F 1$ appeared in our model (29) and in physical quantities $\rho, p$ and $\Lambda$. The Barber's scalar function $\phi(t)$ is to be introduced in the field equations in order to have gravitational parameter $G$, a function of $t$ where as in Einstein theory it is a constant. The whole properties of the universe affected by $\phi(t)$ and it yield the model of the universe in more than three spatial dimensions, since the Gauss hypergeometric function $2 F 1$ is appeared in it. So that our model (29) describe the universe of more than three spatial dimensions i.e., four, five and so on.

The geometrical and physical features of our model (29) in more than three spatial dimensions describes by $\rho, p, \Lambda$ and $\phi$. The Barber's scalar function $\phi(t)$ is diverges to minus infinity at early stage of universe and goes on increasing when time $t$ increasing and diverges to infinity at final stage of universe, involving Gauss hypergeometric function $2 F 1$. In view of nature of $\phi(t)$, it is observed that the cosmological term $\Lambda$ is very very large and tends to infinity at early time and yields a small positive value at late epoch of time. The density $\rho$ and pressure $p$ of the fluid diverges to infinity at the early stage of the universe and they goes on decreasing when time $t$ increases and attain the constant value when $t \rightarrow \infty$, though the barber scalar function $\phi(t)$ present in it. The proposed variation law for Hubble parameters leads the cosmological model (29) in which initial stage is dominated by decelerating phase and at late time universe expanding with acceleration. The model has shear and expansion and in the beginning they are very very large and then they gradually decreases and there is no shear at final stage of universe and expansion attain the value ' $3 a$ ' at late epoch of time. The model starts with the deceleration expansion and then it is expanding exponentially with acceleration at late time supporting the observational data ${ }^{[19-22]}$. The spatial volume $V$ is zero at initial stage $t=0$ and then it goes on increasing with increase in time $t$, even though the Gauss hypergeometric function $2 F 1$ present in it and finally tends to infinity, when $t \rightarrow \infty$. The limit $\frac{\sigma}{\theta}=0$ as $t \rightarrow \infty$ suggested that the model is isotropizes for large value of $t$. The coefficient of bulk viscosity $\zeta$ is taken to be constant and does not affect the nature of matter density $\rho$, pressure $p$ and cosmological term $\Lambda$. When $t \rightarrow 0,2 F 1 \rightarrow \infty$ and $2 F 1 \rightarrow 1$ when $t \rightarrow \infty$, for all $n>1$. This suggested that in the beginning of the universe, the model has a spatial geometry of more than three dimensions and then it switched over to three-dimensional spatial geometry at late epoch of time.

It is interesting to note that, the Barber's scalar function $\phi(t)$ play the important role in the behavior of the model of the universe. It is noticed that Gauss hyper-geometric function $2 F 1$ is present in our model (29) which shows that our model describe the universe which has geometry of more than three spatial-dimension in the beginning. This is one of the important point in the geometry of the space-time that the space-time have more than three spatial dimensions, which is the theoretical evidence pointed out in the geometry of universe in this model.

\section{Our Findings}

1. We have investigated Bianchi type I bulk viscous barotropic fluid cosmological model with varying - $\Lambda$ and functional relation on Hubble parameter in self-creation theory of gravitation.

2. The Gauss hyper-geometric function $2 F 1$ is present in our model (29) which shows that our model of universe may have more than three spatial-dimensions in the beginning which is the theoretical evidence pointed out in the geometry of universe in this model.

3. The Barber's scalar function $\phi(t)$ diverges to minus infinity at early stage of the universe and goes on increasing, when a time $t$ increases and diverges to infinity at final stage of universe, involving Gauss hyper-geometric function $2 F 1$.

4. The Barber's scalar function $\phi(t)$ is appeared in energy density $\rho$, pressure $p$ and cosmological constant $\Lambda$ with Gauss hypergeometric function $2 F 1$ and involved in the nature of it. At early stage of universe, the density $\rho$ and pressure $p$ diverges to infinity and they attain constant values at late epoch of time. This shows that the model filled with matter with uniform density at late epoch of time.

5. In view of the behavior of $\phi(t)$, it is observed that the cosmological term $\Lambda$ is very very large in the beginning and has small positive value at late epoch of time.

6. The propose variation law for Hubble parameter leads cosmological model in which initial stage is dominated by decelerating phase of the universe and at later stage, the universe has accelerating phase and the universe is expanding exponentially with acceleration supporting the observational data ${ }^{[19-22]}$. 
7. The model has shear and expansion and in the beginning they are very very large and tends to infinity and then they gradually decreases and at final stage, there is no shear in the universe and expansion attain the value ' $3 a$ ' at late epoch of time, shows uniform expansion of the universe at late epoch of time.

8. The spatial volume $V$ is zero at initial stage $t=0$ and then it goes on increasing with increase in time $t$, even though the Gauss hypergeometric function $2 F 1$ present in it and finally tends to infinity, when $t \rightarrow \infty$.

9. When $t \rightarrow 0,2 F 1 \rightarrow \infty$ and $2 F 1 \rightarrow 1$ when $t \rightarrow \infty$, for all $n>1$. This suggested that the universe has a geometry of more than three spatial-dimensions at early stage of universe and then it switched over to three-dimensional spatial geometry at late epoch of time.

10. The coefficient of bulk viscosity $\zeta$ is taken to be constant and does not affect the nature of density $\rho$, pressure $p$ and cosmological term $\Lambda$.

11. The limit $\frac{\sigma}{\theta}=0$ as $t \rightarrow \infty$ suggested that the model having isotropic nature for large value of $t$.

\section{Conclusion}

We have investigated Bianchi type I bulk viscous barotropic fluid cosmological model with varying $\Lambda$ and functional relation on Hubble parameter in self-creation theory of gravitation and it is seen that our model may have more than three spatial-dimensions in the beginning and then it switched over to three-dimensional spatial geometry at late epoch of time. This is theoretical evidence pointed out in this model that the universe has more than three spatial-dimensions in the beginning. This is happening because of the presence of Barber's scalar function $\phi(t)$. The model has exponentially accelerating expansion at late time starting with decelerating expansion which supported the observation ${ }^{[19-22]}$. The other geometrical and physical aspects of the model have been studied which are given in findings.

\section{Acknowledgement}

The authors are very much grateful to UGC, New Delhi for supporting financial assistance under Major Research Project for this work.

\section{References}

[1] G. A. Barber, On two "Self-Creation Cosmology", Gen. Rel. Grav., vol. 14, pp. 117- 136, (1982).

[2] L. O. Pimentel, "Exact self-creation cosmological solutions", Astrophys. Space Sci.,vol. 116, pp. 395-399, (1985).

[3] H. H. Soleng, "Self-creation cosmological solutions",
Astrophys. Space Sci., Vol. 139, pp. 13-19, (1987).

[4] D. R. K. Reddy and R. Venkateswarlu, "Bianchi type-VI0 models in self-creation Cosmology", Astrophys. Space Sci., vol. 155, pp. 135-139, (1989).

[5] R. Venkateswarlu and D. R. K. Reddy, "Bianchi type-I models in self -creation theory of gravitation", Astrophys. Space Sci., vol. 168, pp. 193-199, (1990).

[6] K. Shanti and V. U. M. Rao, "Bianchi type-II and III models in self- Creation cosmology", Astrophys Space Sci., vol. 179, pp. 147-153 (1991).

[7] V. U. M. Rao and Y. V. S. S. Sanyasiraju, "Exact Bianchi-type VIII and IX models in the presence of zero-mass scalar fields", Astrophys. Space Sci., vol. 187, pp. 113-117, (1992).

[8] S. Ram and C. P. Singh, "Early universe in self-creation cosmology", Astrophys. Space Sci., vol. 257, pp. 123-129 (1997).

[9] A. Pradhan and H. R. Pandey, "Bulk viscous cosmological models in Barber's second self- creation theory", Indian J. Pure Appl. Math., vol. 35, pp. 513-523, (2004).

[10] U. K. Panigrahi and R. C. Sahu, "Plane symmetric cosmological micro model in modified theory of Einstein's general relativity", Theoret. Appl. Mech., vol. 30, pp. 163-175, (2003).

[11] D. R. K. Reddy, "Plane symmetric string cosmological models in self-creation theory of gravitation", Astrophys Space Sci., vol. 305, pp. 139-141, (2006).

[12] D. R. K. Reddy et al., "Axially symmetric cosmic strings in a scalar-tensor theory”, Astrophys space sci., vol. 306, pp. 185-188, (2006).

[13] R. Venkateswarlu et al.: String cosmological solutions in self-creation theory of gravitation, Int.J.Theor.Phys., vol. 47, pp. 640-648, (2008).

[14] V. U. M. Rao et al., "Exact Bianchi type II, VIII and IX string cosmological models in Saez- Ballester theory of gravitation", Astrophys Space Sci., vol. 314, pp. 73-77, (2008).

[15] V. U. M. Rao and T. Vinutha, "Plane symmetric string cosmological models in self-creation theory of gravitation", Astrophys Space Sci., vol. 325, pp. 59-62, (2010).

[16] G. S. Rathore and K. Mandawat, "Five dimensional perfect fluid cosmological models in Barber's second self-creation theory", Adv. Studies Theor. Phys., vol. 4, pp. 213-224, (2010).

[17] S. D. Katore et al., "Bianchi type-III cosmic strings and domain walls cosmological models in self-creation cosmology", Prespacetime Journal, vol. 2, pp. 447-461, (2011).

[18] Borkar M. S. and Ashtankar N. K., "Plane symmetric viscous fluid cosmological model with varying $\Lambda$-term in self creation theory of gravitation", Tensor N. S., vol. 73, pp. 228, (2013).

[19] Perlmutter, S., et al., "Discovery of supernova explosion at half the age of the Universe", Nature, vol. 391, pp. 51-54, (1998).

[20] Knop, R. A., et al., Astrophys. J. vol. 598, pp. 102 (2003) 
[21] Tegmark, M., et al., Phys. Rev. D 69, pp. 103-501 (2004)
[22] Spergel, D. N., et al., WMAP three years results: implication for cosmology, Preprint astro-ph/0603449, (2006). 